Revista Digital Universitaria

Vol. 22, Núm. 6, noviembre-diciembre 2021

\title{
La pigmentación en la piel humana como adaptación al ambiente
}

Antonio T. Araujo Soto

\section{Resumen}

La diversidad biológica y cultural que caracteriza a la humanidad es un reflejo de su historia evolutiva en el planeta. No obstante, esta variedad ha sido repetidamente utilizada como instrumento de marginación y discriminación entre grupos humanos, que no consideran los distintos entornos en los cuales se ha desenvuelto nuestra especie y que favorecieron la diversidad que observamos. En este trabajo, se revisan algunas investigaciones sobre la pigmentación de la piel en los humanos. Distintas líneas de estudio muestran que la variedad en la pigmentación de la dermis se debe, particularmente, a diferencias en la exposición a la radiación uv y sus efectos en el metabolismo humano, así como en su reproducción. Igualmente, la evidencia indica que poblaciones humanas ubicadas cerca del ecuador requirieron de una mayor pigmentación (melanina) para proteger el ADN y otras biomoléculas de los efectos dañinos de la radiación solar; mientras que, en latitudes con menor exposición de radiación uv, la piel con una menor cantidad de pigmento o melanina facilitó la síntesis de vitamina D en poblaciones establecidas hacia el norte del ecuador.

Palabras clave: evolución, melanina, pigmentación, adaptación, discriminación.

\section{HUMAN SKIN PIGMENTATION AS AN ENVIRONMENT ADAPTATION}

\begin{abstract}
The evolutionary history of humankind is a reflection of its biological and cultural diversity. However, such variety has been repeatedly used as an instrument for marginalization and discrimination amongst people, who do not consider the human species-developing circumstances that have favored our current diversity. In the present work, we review some research about skin pigmentation. A range of studies shows that dermis pigmentation variety is caused by differences in uv-radiation exposure and its effects on human metabolism and on reproduction. Likewise, evidence indicates that people who live in regions near the equator required increased pigmentation (melanin production) to protect DNA and other biomolecules from the harmful effects of solar radiation; while in latitudes with less uv-radiation exposure, lightly pigmented skin facilitated vitamin D synthesis in populations established towards the north of the equator.
\end{abstract}

Keywords: evolution, melanin, pigmentation, adaptation, discrimination. 
Biólogo con estudios de posgrado en la Universidad Nacional Autónoma de México UNAm). Ha colaborado en distintos proyectos, al estudiar la variabilidad genética de la población mexicana y el efecto de contaminantes ambientales en poblaciones humanas. Le interesa la interacción ambiente-organismo en un contexto evolutivo y biomédico. Actualmente me desempeña como docente de Bachillerato.

\section{Introducción}

L

a muerte de George Floyd, en mayo de 2020, provocó la indignación de una parte de la sociedad en Estados Unidos y en otras regiones del y resaltar las diferencias que persisten por cuestiones raciales, un tema que acompaña a la humanidad desde hace siglos (BBC News Mundo, 2020).

A través de la historia, la creencia del valor de las personas de acuerdo con su cultura o características físicas se ha formado y fluido al amparo del pensamiento etnocéntrico de sociedades que dominan sobre otras, como el de las naciones colonialistas entre los siglos xv y xIx (Menéndez, 2018, p. 12). No obstante, las conductas, al igual que los saberes, cambian, en la medida en que conocemos e integramos nueva información que nos ayuda a entender el mundo. Por ejemplo, la pigmentación de la piel, que continúa usándose como motivo de discriminación, es evidencia cierta y significativa de la adaptación a las condiciones del entorno en el cual se han desarrollado distintos grupos humanos.

En el transcurso del siglo xx, diferentes investigaciones han contribuido a revelar los mecanismos moleculares de la variación en la pigmentación de la piel y el papel del ambiente como factor de su diversidad. Además, el estudio de nuestro ADN evidencia el fondo genético subyacente de este proceso adaptativo, que tiene miles de años y que se ha desarrollado de forma independiente en distintas poblaciones (Deng y Xu, 2018). 


\section{Melanina y su efecto protector}

La melanina es un conjunto de pigmentos que se encuentran en distintos organismos, incluyendo bacterias, plantas, hongos y animales. Los pigmentos se obtienen a partir de diferentes sustratos químicos y entre sus funciones está la protección en contra de la radiación ultravioleta (uv). Esto se debe a su capacidad de absorción del espectro electromagnético, además de su alto índice de refracción - la facultad de disminuir la velocidad con que incide la radiación que proviene del sol cuando toca un organismo (D'Alba y Shawkey, 2019, pp. 1-2).

La pigmentación de la piel en todos los humanos se debe a la melanina, que se compone predominantemente de dos tipos de macromoléculas: la eumelanina, de color marrón-oscuro, y la feomelanina, que es rojo-amarillo. Estos pigmentos se forman durante un proceso conocido como melanogénesis, a partir de la oxidación del aminoácido tirosina, en una reacción mediada por la enzima tirosinasa (TYR).

La melanina se produce en estructuras celulares especializadas que llamamos melanosomas, los cuales se encuentran en los melanocitos, unas células ubicadas en el estrato basal de la epidermis (capa superficial de la piel), así como en otras partes del cuerpo. Después de la síntesis y almacenamiento del pigmento, los melanosomas son trasportados a través de prolongaciones celulares hasta los queratinocitos, el principal componente celular de la piel (ver figura 1). En los queratinocitos, los melanosomas se disponen cerca del núcleo, lo que los protege de los efectos dañinos de la radiación uv, que es absorbida y disipada en forma de calor. La melanina también puede actuar como antioxidante, deteniendo los radicales libres que se forman bajo la acción de la radiación (Swope y Abdel-Malek, 2018, p. 2; Nasti y Timares, 2014, pp. 188-189).

Figura 1. La melanina se sintetiza en los melanosomas, los cuales son transportados de los melanocitos hacia los queratinocitos, por medio de prolongaciones celulares. En los melanosomas se sintetizan las dos moléculas que conforman la melanina (eumelanina y la feomelanina). La relación entre ambas es determinada por la disponibilidad del aminoácido cisteína que interviene en la producción de feomelanina, así como de la expresión de enzimas (TRP-1, TRP-2) necesarias en la producción de la eumelanina (imagen modificada de Sturm et al.,

1998).

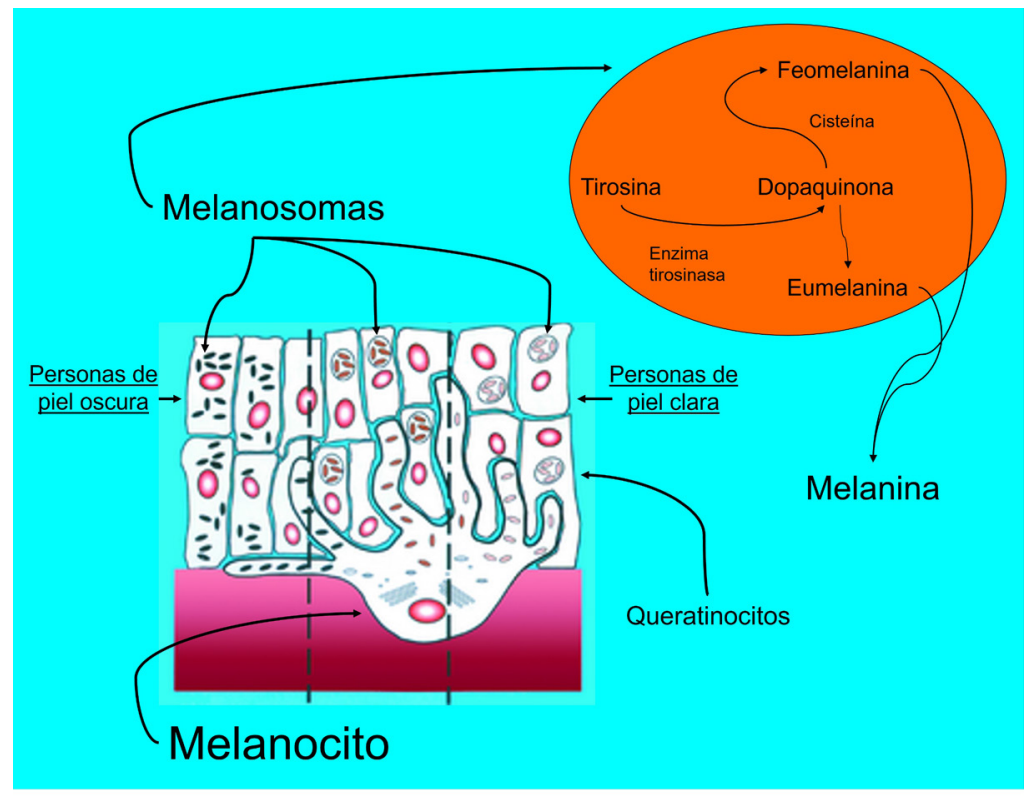

Los melanocitos sintetizan las dos biomoléculas (eumelanina y feomelanina) que conforman la melanina, pero el efecto protector depende principalmente de 
Figura 2. Visualización de la melanina en distintos tipos de piel humana (A: muy clara; F: oscura). La melanina se observa de color negro en la epidermis, así como en el estrato basal; en este último, se encuentran los melanocitos, las células que sintetizan el pigmento (imagen adecuada con permiso de Del Bino et al., 2015). la eumelanina. Por lo tanto, la piel con concentraciones menores de eumelanina es más susceptible de sufrir quemaduras por exposición al sol, fotoenvejecimiento y cáncer (Swope y Abdel-Malek, 2018, p. 2; Nasti y Timares, 2014, p. 191).

Las diferencias que observamos en la pigmentación de la piel se deben a varios factores. Fisiológicamente, el número de melanocitos es similar en los diversos grupos humanos, pero la organización de los melanosomas y la concentración de melanina son variables. La piel oscura o que puede experimentar un proceso de bronceado dispone de melanosomas grandes, individuales y abundantes en melanina. En cambio, en la piel muy clara, los orgánulos contienen gránulos de pigmento pequeños, con menor cantidad de melanina y agrupados (ver figura 2). Otro elemento que media en las diferencias que observamos en la pigmentación es la degradación de los melanosomas en los queratinocitos, proceso que ocurre en una tasa mayor en pieles claras (Lambert et al., 2019, pp. 408-409).

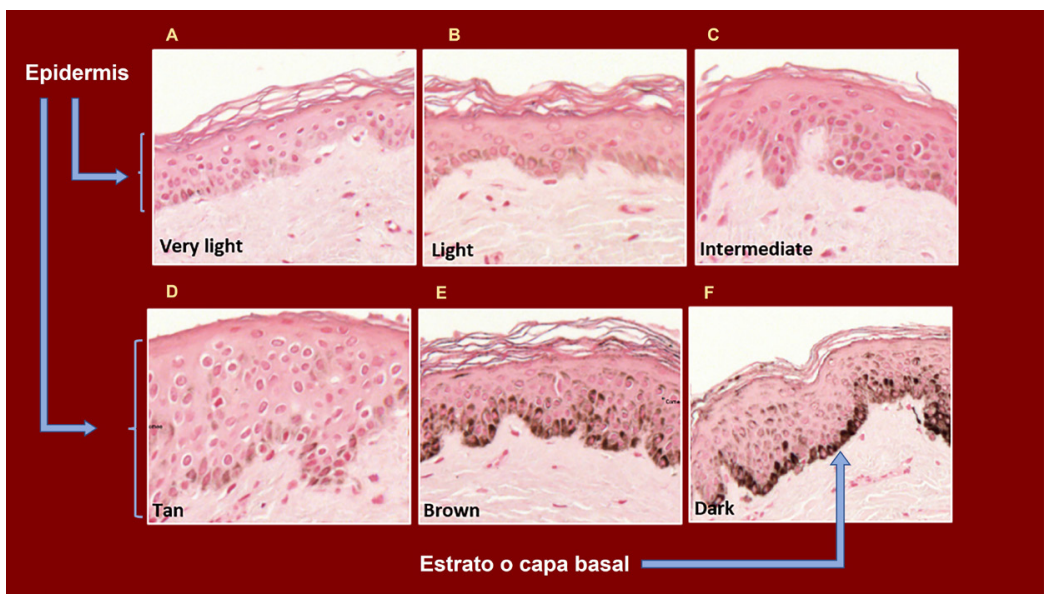

Además de la fisiología intrínseca de nuestra especie, la variación en la pigmentación se encuentra asociada a factores ambientales, especialmente a la radiación uv. Durante mucho tiempo, se ha documentado que la coloración de la epidermis presenta una disposición particular alrededor del planeta, con poblaciones de piel oscurecida que habitaban originalmente regiones cercanas a la línea del ecuador, donde la exposición a la radiación uv es mayor, así como la aridez y el calor. No obstante, el desplazamiento de grupos humanos en los últimos siglos ha modificado esta distribución.

\section{Hipótesis sobre la pigmentación de la piel}

Explicar la variación en la pigmentación ha llevado a plantear varias hipótesis, las cuales difieren sobre la causa de una mayor o menor cantidad de melanina en grupos humanos. Sin embargo, la mayoría señala la importancia del entorno como determinante en la pigmentación, y su función protectora frente a distintos estresores, en particular a los efectos dañinos de la radicación solar. Entre las hipótesis sugeridas se encuentran: protección del cáncer de piel, defensa contra 
Figura 3. Mapa de la pigmentación humana. En regiones cercanas al ecuador se encuentran grupos humanos de piel oscurecida o con mayor pigmentación, mientras que en localidades alejadas se observan poblaciones con menor pigmentación. Las flechas indican un modelo de migración del Homo sapiens fuera del continente africano ("Mapa creado por George Chaplin", uso y modificación con permiso de https://sites.psu.edu/ ninajablonski/). microorganismos, camuflaje, protección y síntesis de biomoléculas, dimorfismo y selección sexual (Juzeniene et al., 2009).

De las distintas propuestas, la protección y síntesis de biomoléculas explica convincentemente la correlación geográfica entre la distribución de radiación uv y la variabilidad de melanina en poblaciones ubicadas en diferentes latitudes, y con ello, la variación en la pigmentación humana (ver figura 3). Este tipo de radiación es un mutágeno, causa de cáncer de piel y un agente asociado a la degradación del nutriente folato, en su forma principal 5-metiltetrahidrofolato.

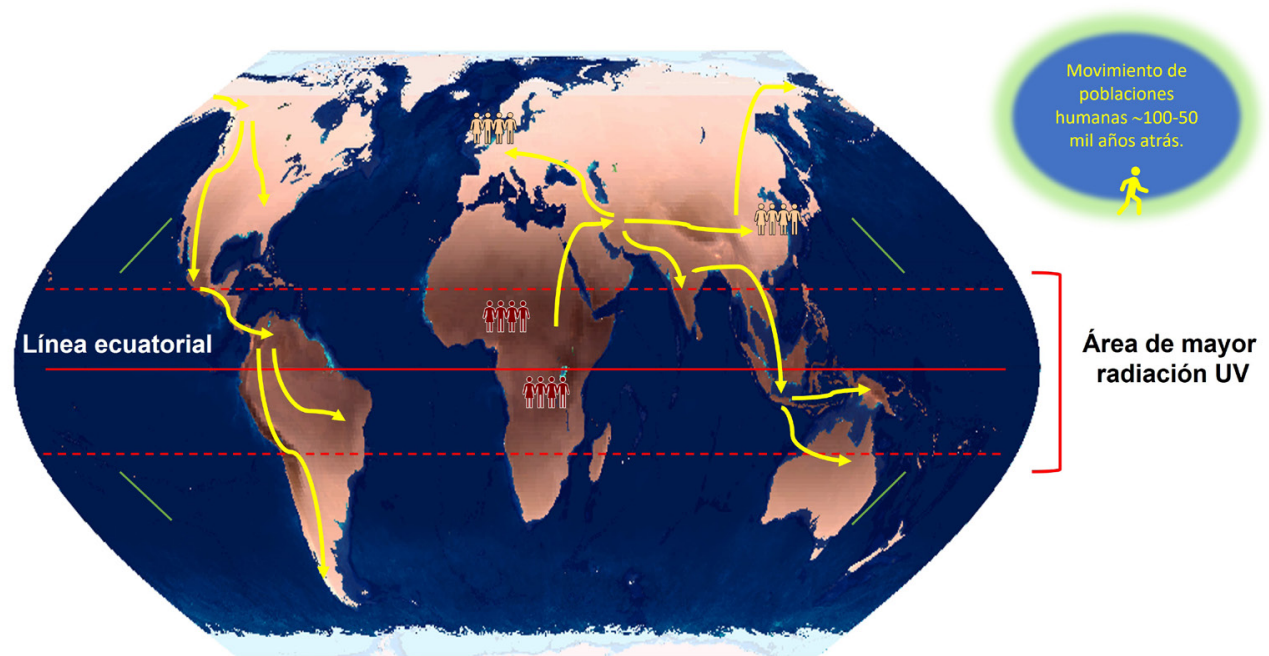

Deficiencias en este nutriente pueden ocasionar problemas en el desarrollo de la columna vertebral (espina bífida) y del encéfalo (anencefalia), dos defectos del nacimiento que ocurren por la incompleta formación del tubo neural en etapas tempranas del desarrollo embrionario (Centros pata el Control y la Prevención de Enfermedades, 2020). También se han reportado problemas de infertilidad masculina. Además, el folato es requerido en la síntesis de purinas, compuestos químicos que forman parte de la estructura del ADN y necesarias durante la reparación y replicación del material genético (Jablonski y Chaplin, 2017; Jones et al., 2018). En conjunto, esta evidencia señala el efecto dañino de la radiación uv en la integridad del ADN y en la reproducción.

De acuerdo con esta hipótesis, en poblaciones antiguas establecidas cerca del ecuador, los efectos dañinos de la radiación se presentaron con menor frecuencia en individuos que sintetizaban más melanina en la piel. Con el tiempo, la reproducción favorable de estos sujetos dio lugar a poblaciones con pigmentación más oscura en estas latitudes. La piel oscura o con más melanina es una protección cuando las personas se encuentran expuestas a altas dosis de radiación uv.

Por otra parte, conforme nuestra especie se desplazó fuera del continente africano, se encontró con nuevas condiciones ambientales, patógenos y fuentes de alimentación, que con el tiempo conducirían al desarrollo de adaptaciones 
locales y aportarían diversidad a la humanidad (Fan et al., 2016). Igualmente, el movimiento de poblaciones humanas hacia latitudes al norte del ecuador supuso un cambio en la pigmentación de la piel en estos grupos. Esta transición significó la reproducción favorable de individuos con menos melanina, un cambio condicionado por la menor exposición a radiación uv que es necesaria para la síntesis de vitamina D.

El cambio en la pigmentación facilitó la síntesis de esta vitamina, proceso que en pieles de tonalidad oscura se habría dificultado debido a la absorción que hace la melanina de la radiación uv, sumado a las bajas cantidades de radiación en latitudes al norte del ecuador. La vitamina D regula los niveles de calcio en las células, y estudios recientes indican que participa en otros procesos de la piel como la diferenciación celular de queratinocitos y en la secreción de proteínas antimicrobianas. Este nutriente se adquiere naturalmente de alimentos como el pescado, el queso o la yema de huevo, pero es a través de la piel expuesta a la luz del sol que la mayoría de las personas sintetizan esta vitamina (Jablonski y Chaplin, 2017; Jones et al., 2018).

Notablemente, las diferencias geográficas que se observan en la pigmentación humana están acompañadas de variantes (polimorfismos) en la secuencia de genes (MC1R, SLC45A, SLC24A5, ASIP, TYR, OCA2, KITLG, entre otros), que en distintas poblaciones se encuentran asociados a una pigmentación más clara, así como a un mayor riesgo a desarrollar distintos tipos de cáncer de piel. Por ejemplo, variantes en el gen MC1R (receptor de melanocortina 1) se encuentran fuertemente asociadas con el cabello de color rojizo, piel clara y la presencia de pecas, además del riesgo a desarrollar melanoma. En distintas poblaciones se pueden encontrar diferentes variantes de un mismo gen, lo que podría indicar procesos de adaptación local y convergencia evolutiva, es decir, el desarrollo de un rasgo compartido entre grupos humanos separados (Nasti y Timares, 2014, p. 191; Deng y Xu, 2018; Swope y Abdel-Malek, 2018, pp. 5-6).

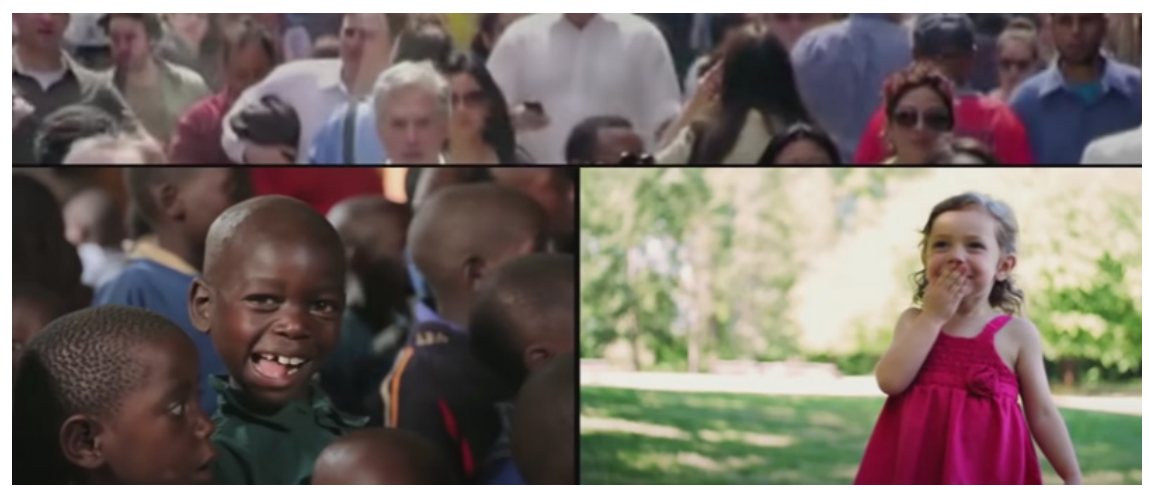

Video. La biología del color de piel (en inglés, con subtítulos en español) (biointeractive, 2015).
Por otra parte, también se han reportado polimorfismos compartidos en distintas poblaciones, lo cual es interpretado como un evento temprano en la evolución de los humanos modernos y que antecedió a la separación de poblaciones durante la migración y asentamiento en diferentes entornos. Un 
ejemplo de lo anterior son algunas variaciones encontradas en el gen KITLG, el cual participa en el desarrollo de los melanocitos en la piel adulta. Estas variaciones se presentan en una alta frecuencia en pobladores europeos y asiáticos, pero no en africanos (Deng y Xu, 2018, p. 3). Sin duda, el análisis genético ha aportado nuevas evidencias y preguntas sobre el proceso de pigmentación en la especie humana.

\section{Conclusiones}

Durante mucho tiempo, las diferencias biológicas y culturales de grupos humanos han sido utilizadas para promover la marginación, desigualdad y discriminación. Ejemplo de ello es la pigmentación de la piel, que sigue siendo el pretexto de prácticas contrarias a los derechos humanos. Sin embargo, el interés por conocer nuestros orígenes y el trabajo multidisciplinario han aportado nuevas evidencias sobre la pigmentación de nuestra especie, que consiste en un proceso complejo, pero explicable a la razón de la biología y en el que debe caber el sano ejercicio del debate científico, pero no la discriminación.

\section{Referencias}

* bBC News Mundo. (2020, 30 de mayo). George Floyd: el dolor y la ira sacuden unas 50 ciudades de EE.UU. tras la muerte del hombre afroestadounidense bajo custodia policial. https://cutt.ly/KRzKWhG

* biointeractive. (2015, 20 de julio). The Biology of Skin Color - HHMI Biolnteractive Video [video]. YouTube. https://youtu.be/hFw8mMzH5YA

* D’Alba, L. y Shawkey, M. D. (2019). Melanosomes: biogenesis, properties, and evolution of an ancient organelle. Physiological Reviews, 99(1), 1-19. https://doi. org/10.1152/physrev.00059.2017

- Del Bino, S., Ito, S., Sok, J., Nakanishi, Y., Bastien, P., Wakamatsu, K. y Bernerd, F. (2015, noviembre). Chemical analysis of constitutive pigmentation of human epidermis reveals constant eumelanin to pheomelanin ratio. Pigment Cell \& Melanoma Research, 28(6), 707-717. https://doi.org/10.1111/pcmr.12410

* Deng, L. y Xu, S. (2018). Adaptation of human skin color in various populations. Hereditas, 155(1). https://doi.org/10.1186/s41065-017-0036-2

* Centros para el Control y la Prevención de Enfermedades (2020, 1 de julio). Conceptos básicos sobre el ácido fólico. Ácido fólico. https://www.cdc.gov/ncbddd/ spanish/folicacid/about.html

* Fan, S., Hansen, M. E., Lo, Y. y Tishkoff, S. A. (2016). Going global by adapting local: a review of recent human adaptation. Science, 354(6308), 54-59. https://doi. org/10.1126/science.aaf5098

* Jablonski, N. G. y Chaplin, G. (2017). The colours of humanity: the evolution of pigmentation in the human lineage. Philosophical transactions of the Royal Society of London. Series B, 372(1724): 20160349. https://doi.org/10.1098/rstb.2016.0349 
* Jones, P., Lucock, M., Vaysey, M.y Beckett, E. (2018). The vitamin D-Folate hypothesis as an evolutionary model for skin pigmentation: an update and integration of current ideas. Nutrients, 10(5), 554. https://doi.org/10.3390/nu10050554

* Juzeniene, A., Setlow, R., Porojnicu, A., Steindal, A. H.y Moan, J. (2009). Development of different human skin colors: A review highlighting photobiological and photobiophysical aspects. Journal of Photochemistry and Photobiology B: Biology, 96(2): 93-100. https://doi.org/10.1016/j.jphotobiol.2009.04.009.

* Lambert, M. W., Maddukuri, S., Karanfilian, K. M., Elias, M. L. y Lambert, W. C. (2019). The physiology of melanin deposition in health and disease. Clinics in Dermatology, 37(5), 402-417. https://doi.org/10.1016/j.clindermatol.2019.07.013

* Menéndez, E. L. (2018). Colonialismo, neocolonialismo y racismo: el papel de la ideología y de la ciencia en las estrategias de control y dominación. Universidad Nacional Autónoma de México, Programa Universitario de Estudios de la Diversidad Cultural y la Interculturalidad. https://cutt.ly/pRzBKEf

* Nasti, T. H. y Timares, L. (2014). MC1R, eumelanin and Pheomelanin: Their role in determining the susceptibility to skin cancer. Photochemistry and Photobiology, 91(1), 188-200. https://doi.org/10.1111/php.12335

* Sturm, R. A., Box, N. F. y Ramsay, M. (1998). Human pigmentation genetics: the difference is only skin deep. BioEssays, 20(9), 712-721. https://cutt.ly/jRxgPpM

* Swope, V. B. y Abdel-Malek, Z. A. (2018). MC1R: Front and center in the bright side of dark eumelanin and DNA repair. International Journal of Molecular Sciences, 19(9), 2667. https://doi.org/10.3390/ijms19092667

\section{Cómo citar este artículo}

* Araujo Soto, Antonio T. (2021, noviembre-diciembre). La pigmentación en la piel humana como adaptación al ambiente. Revista Digital Universitaria (RDU), 22(6). http://doi.org/10.22201/cuaieed.16076079e.2021.22.6.2 